\title{
JULIÁN DEL REY: NUEVOS DATOS SOBRE SU FIGURA
}

\author{
POR
}

\author{
GERMÁN DUEÑAS BERAIZ
}

\section{RESUMEN - ABSTRACT}

Los artesanos hispanos gozaron de gran reputación durante la Edad Moderna a la hora de la fabricación de espadas. Uno de los espaderos más conocidos fue Julián del Rey. A pesar de ello tenemos pocas y confusas noticias sobre su vida y producción. Después de repasar lo que hasta el momento se sabía sobre él, se aportan una serie de datos novedosos procedentes de un documento hasta ahora desconocido entre los especialistas.

The spanish swordsmiths were very reputed in the Modern Age. Julián del Rey was one of most important swordsmiths, however we have confuse news about his life and production. This paper provides new information on his figure through an unknown document

\section{PALABRAS CLAVE - KEY WORDS}

Fabricación de espadas. Marcas. Zaragoza. Julián del Rey. Siglo XVI.

Swords manufacture. Trademarks. Zaragoza. Julián del Rey. Sixteenth century.

\section{INTRODUCCIÓN}

Muchas de las armas que se fabricaron durante la Edad Moderna en la Península Ibérica gozaron de gran fama en toda Europa, aunque si tuviéramos que destacar alguna en concreto, es muy posible que mencionáramos la realización de todo tipo de espadas.

Esta producción de carácter artesanal dio lugar a toda una serie de figuras de renombre que destacaron por sus conocimientos en la materia, especialmente en la forja y temple de las hojas. Son muchos los espaderos de los que tenemos noticias, aunque éstas sólo se suelen consignar al nombre y la marca. Y de los que además, existen numerosas piezas repartidas en diferentes Museos y colecciones particulares por todo el mundo, dando fe de la existencia y el dominio que de su oficio tenían dichos maestros.

Entre todos ellos sobresale un pequeño grupo cuyo renombre ha llegado hasta nuestros días. Uno de los más destacados fue Julián del Rey. Fruto de ese prestigio, su nombre fue mencionado siempre en los diferentes libros y artículos en los que se hablaba de la producción de espadas en la monarquía hispana.

Las escasas noticias que aparecen recogidas en estas obras, dan sin embargo como resultado un conjunto de datos inconexos y muchas veces contradictorios sobre su figura que no permiten elaborar un relato mínimo sobre él. Por ello se ha creado una imagen rodeada de misterio, ocasionando que muchas veces se confundiera la realidad histórica con la simple leyenda. Este rasgo no es exclusivo de Julián del Rey, y se da en muchos de los artesanos 
que en las diferentes disciplinas de la construcción de armas existieron en España. Pero quizás si es uno de los más destacados en este aspecto.

Cabe entonces realizarnos una serie de preguntas ¿dónde radica el atractivo de Julián del Rey?; ¿qué lo diferencia del resto de los espaderos?; ¿qué sabemos exactamente sobre él?; y ¿de dónde proceden esos datos? Para resolver todas estas preguntas se hace necesario remontarnos a lo dicho sobre él en el pasado.

\section{ESTADO DE LA CUESTIÓN}

Las cuestiones polémicas que a este personaje rodean se centran en tres temas: la localización geográfica, su cronología y el tema de su marca.

El primer autor que hace referencia a él es Francisco Javier Santiago y Palomares. Este escribano toledano realizó un trabajo en 1762, donde pretendió reunir los nombres y las marcas de todos los espaderos toledanos que trabajaron en su época de mayor esplendor ${ }^{1}$.

El trabajo constaba de dos páginas. En la primera aparecían numerados los nombres, en algunas ocasiones junto con algún dato más de estos espaderos. Mientras que en la segunda, figuraban sus marcas junto con el número al que correspondía.

Palomares nos da aquí los primeros, aunque escasos, datos sobre este maestro espadero. Éstos figuran tras el nombre del espadero, y se refieren a su marca cuando dice uso otras, aunque no especifica cuales, y a las ciudades donde trabajó, al señalar que además de en Toledo Labró también en Zaragoza.

El punzón que le atribuye es el número «59» de la nómina. Concretamente se trata de una figura zoomorfa que se encuentra levantada sobre sus cuartos traseros. Las patas delanteras están suspendidas en el aire en actitud semierguida con la derecha más levantada respecto de la izquierda, mientras que el rabo figura erguido ${ }^{2}$.

La obra de Palomares fue, tanto para éste como para el resto de los espaderos hispanos, la principal referencia que se ha utilizado a la hora de conocer los nombres y las marcas que utilizaron estos artesanos. Este documento de 1762 no alcanzó una verdadera difusión hasta 1849, año en el que fue publicado por Martínez del Romero en el Catálogo de la Real Armería de Madrid ${ }^{3}$.

Poco tiempo después fue Edouard de Beaumont ${ }^{4}$ quien proporcionó nuevos y mayores datos sobre él al comentar la espada jineta nazarí de la Biblioteca Nacional de París ${ }^{5}$, marcada al parecer con un perro. Tal vez sea éste el motivo por el que considera a Julián del Rey como un espadero granadino, ya que a él le atribuye la citada jineta por ostentar como marca dicho animal.

Presumiblemente Beaumont habría estudiado el documento de Palomares, y a pesar de que ambas marcas son claramente diferentes las consideró hechas por el mismo espadero. Pero quizás lo más importante sea la historia con la que glosa la figura de nuestro espadero, ya que al situarlo en Granada como autor de jinetas lo considera un espadero al servicio de Boabdil. Narra cómo tras la conquista del reino nazarí, y debido a su condición de afamado artesano se convirtió al cristianismo de la mano de Fernando el Católico. Este fue el motivo

1 Nomina de los ultimos, y mas famosos armeros de Toledo, que labraron Espadas hasta la entrada del presente Siglo XVIII En que acabó esta Fabrica.

2 El Conde viudo de Valencia de Don Juan en su catálogo de la Real Armería, la considera como una cabra; mientras que el artista que reflejó la nómina de Palomares en el techo de la Sala de Armas de la planta de infantería del Museo del Ejército, lo interpretó como un ciervo de hermosa cornamenta.

3 Martínez Romero, 1849.

4 Beaumont, 1885.

5 París. Bibliotheque Nationale. Cabinet des Medailles Luynes. Inv. 959. 
que le llevó a adoptar el nombre de Julián del Rey6. Por lo tanto aquí encontramos la primera referencia a su origen árabe. Exactamente en la Granada de 1492.

Será en 1890 cuando otro francés, Maindron ${ }^{7}$, levantará las primeras dudas sobre lo que hasta entonces se había dicho sobre Julián del Rey, especialmente en el tema de su marca. Canaliza estas críticas a través de los conocimientos que sobre la cultura islámica poseía Mr. Babelon. Según él era imposible que un árabe, a pesar de haberse cristianizado, adoptara la figura de un perro como marca por considerarse a este animal como un ser impuro en la cultura islámica.

A finales del siglo XIX y principios del XX fueron el Conde Viudo de Valencia de Don Juan y el Barón de Vega de Hoz, sobre todo éste último, quienes trataron la historia de este personaje.

Leguina $^{8}$, en un primer momento, recogió claramente lo dicho por Beaumont, considerándolo como un artesano de origen árabe que floreció en el siglo XV apadrinado por el rey Católico, e identificando su marca con un perro. En una obra posterior ${ }^{9}$ aportó más datos, situándolo en las ciudades de Toledo y Zaragoza, tal y como lo había hecho Palomares, y retrasó su cronología hasta 1478, quizás para hacerla coincidir con la del Rey Chico. En esta ocasión, mencionó además otras marcas usadas por este artesano: la media luna, el mundo con la cruz y una elipse entre dos aspas ${ }^{10}$.

Por otra parte, y por primera vez cita la figura de un descendiente de Julián del Rey. Éste, del mismo nombre, habría trabajado en Toledo y en Valencia y sus marcas habrían sido una cruz de Borgoña o una corona, ambas inscritas.

Tras los datos aportados por Leguina, se identifican presuntamente dos espaderos conocidos como Julián del Rey, y que serían padre e hijo. Su cronología abarcaría desde 1478 hasta la primera mitad del siglo XVI. En cuanto a las ciudades ambos aparecen en Granada, Toledo, Valencia y Zaragoza. Siendo todavía mayor el número de marcas que se les atribuyen llegando a alcanzar la cifra de seis, cuatro para el padre y dos para el hijo.

Ambas cifras se nos antojan excesivas. Un artesano tan afamado no tenía necesidad de desplazarse tanto por la península, a pesar de su condición de morisco, si es que efectivamente lo fue. Respecto a la marca, un espadero tenía en ella el símbolo de su prestigio. Por tanto no le interesaba cambiarla frecuentemente, y aún menos tener varias, ya que en ambos casos provocaría la confusión y el desconcierto entre sus potenciales clientes.

Por tanto, se hace necesaria una revisión crítica de lo que hasta ahora se ha escrito sobre este artesano.

\section{EL PLEITO DE 1549}

Las primeras, aunque débiles, conexiones entre este espadero y la ciudad en la que aparece este documento, Zaragoza, las encontramos en las menciones que se hace a Julián del Rey como el moro de Zaragoza ${ }^{11}$, o al citado comentario de Palomares sobre que labro tambien en Zaragoza ${ }^{12}$.

6 Toda esta teoría no la fundamenta con datos concretos, y pensamos que se trató más bien del fruto del espíritu romántico del autor y de la época.

7 Maindron, 1890

8 Leguina, 1897: 143.

9 Leguina, 1912.

10 Pensamos que el motivo de considerarlas todas ellas como pertenecientes a nuestro espadero, se debía a que aparecieran en hojas que tendrían un perrillo en la hoja. Aunque debido a que no menciona la fuente de esos datos sólo podemos especular sobre ello.

11 Valencia de Don Juan, 1898: 194.

12 Palomares. Op. Cit. 


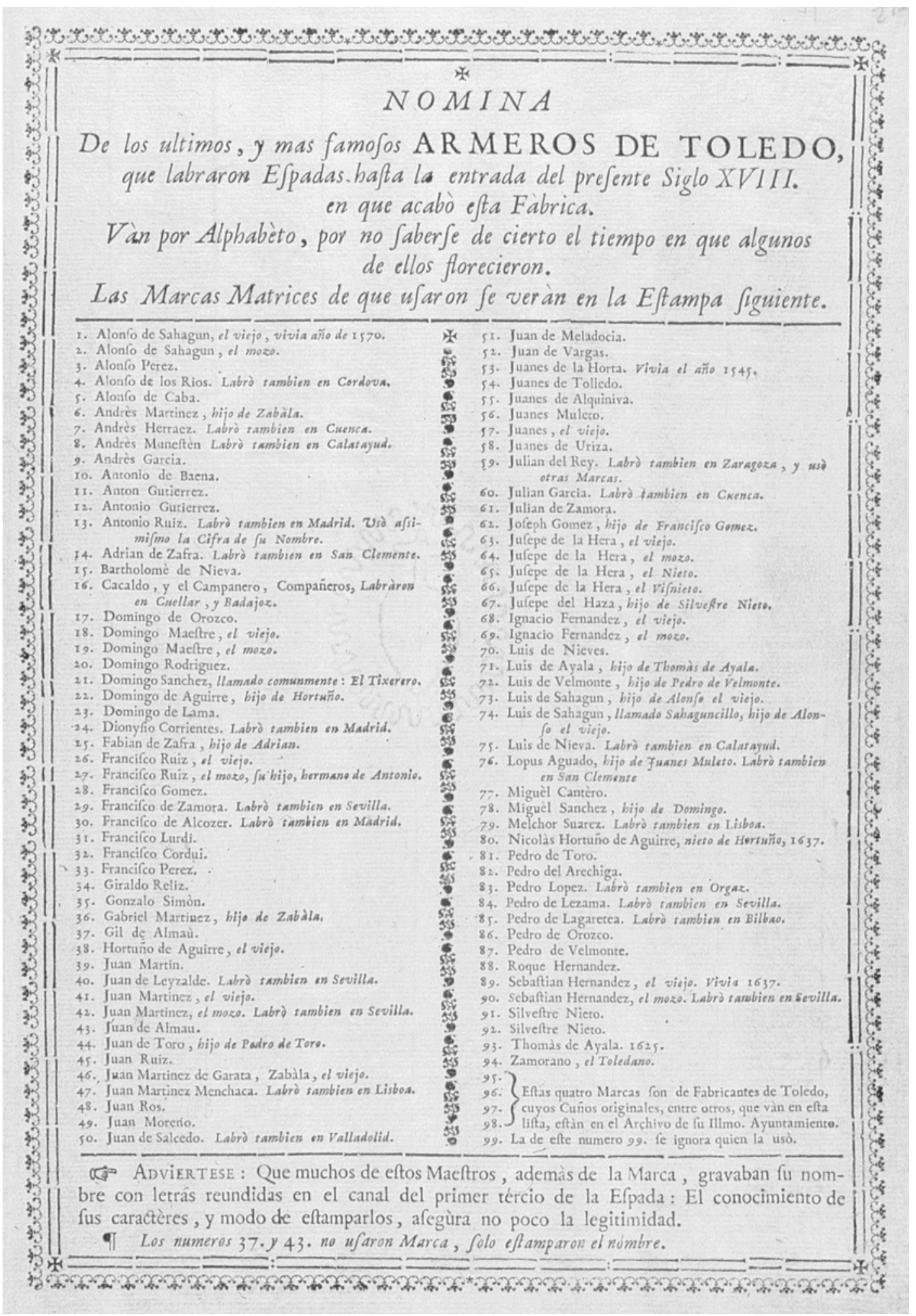

Fig. 1a. Nómina de Palomares en la que recogió los presuntos nombres y marcas de los espaderos que trabajaron en Toledo hasta el siglo XVIII. 


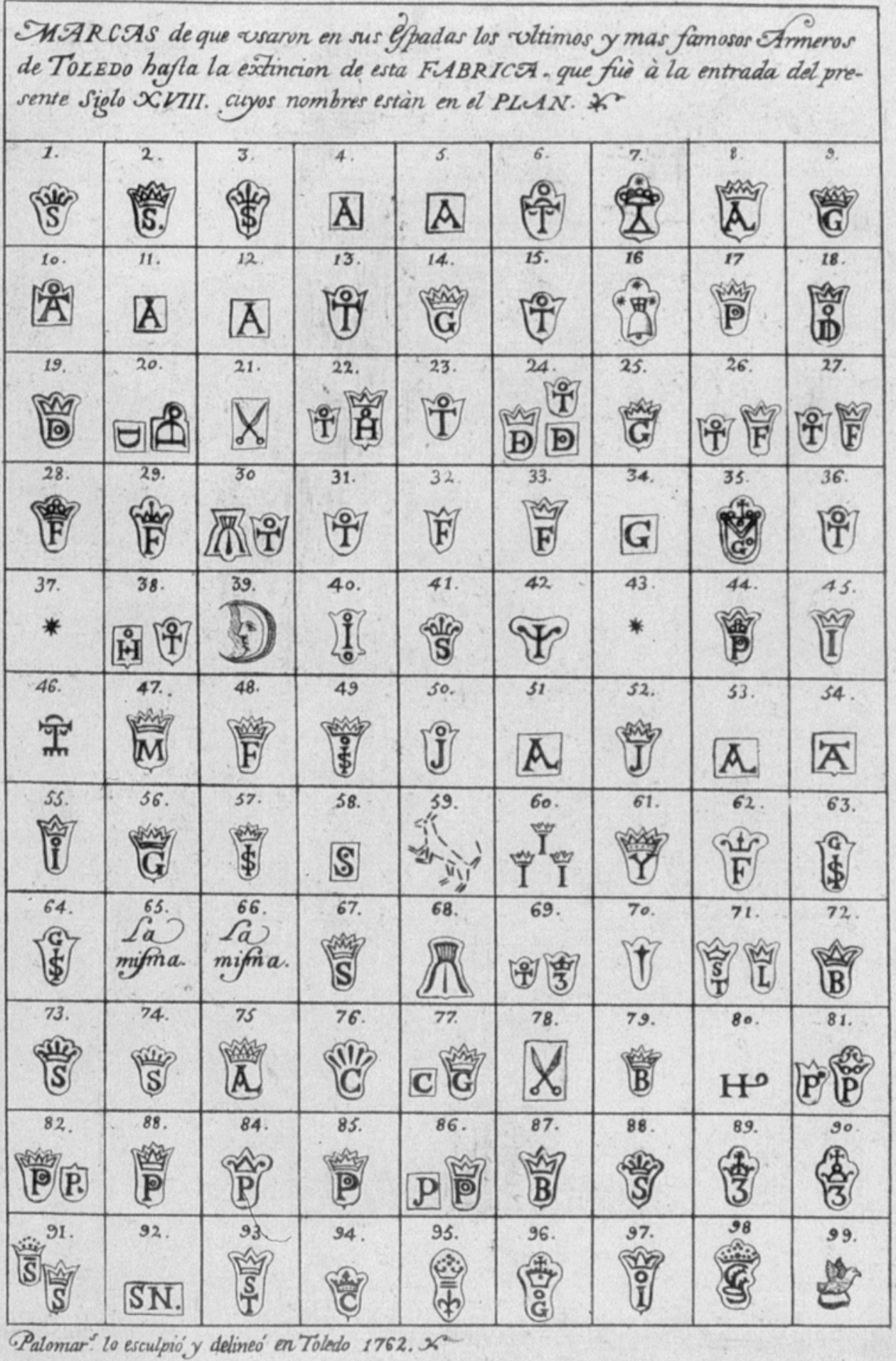

Fig. 1b. Nómina de Palomares en la que recogió los presuntos nombres y marcas de los espaderos que trabajaron en Toledo hasta el siglo XVIII. 
Antes incluso de la localización del documento que nos ocupa, la presencia de Julián del Rey en la ribera del Ebro ya había sido citada con más o menos claridad. El maestro Espés, según señala Asso ${ }^{13}$, indica que Juan II, Arzobispo de Zaragoza, regaló al Rey de Francia, Francisco I (1494-1547), unas espadas muy valoradas entre las que estaban las del morillo, que era una de las formas de denominar a este espadero.

Esto no indica necesariamente que este morillo fuera Julián del Rey, aunque de serlo, es probable que trabajara en Zaragoza, ya que parece lógico pensar que se usaran productos creados en la misma urbe para realizar tales regalos. Por lo tanto podemos decir, aunque con reservas, que existía un espadero en Zaragoza conocido como el morillo. Francisco I murió en 1547, por lo tanto sería en la primera mitad del XVI cuando este morillo trabajó en Zaragoza fabricando espadas.

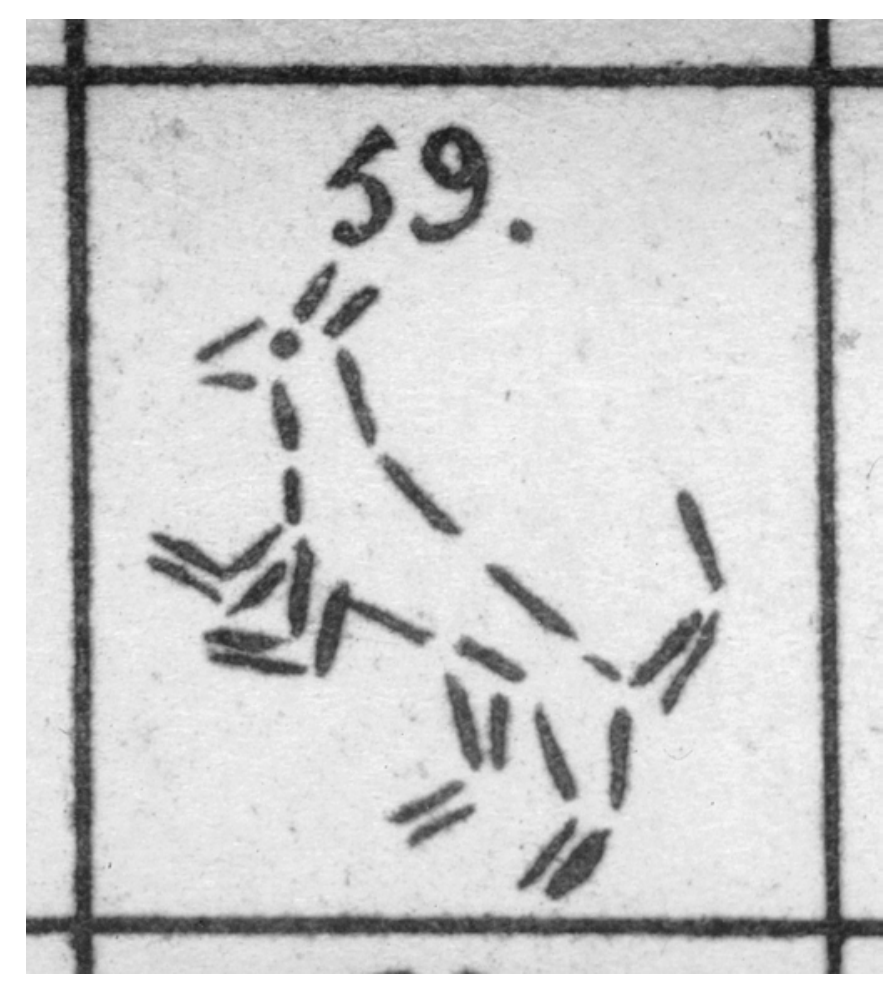

Fig. 2. Detalle de la marca que Palomares atribuye a Julián del Rey, conocida después como del perrillo.

Más clara es la mención que a este artesano hace Ximenez de Embun ${ }^{14}$ al intentar demostrar la existencia de fabricantes de espadas durante el siglo XVI en tierras aragonesas. Este autor cita a un tal Julián del Rey, quien aparece entre la documentación del municipio zaragozano. Este documento es recogido y transcrito posteriormente por Angel San Vicente ${ }^{15}$, y es el que nos sirve para vertebrar el presente artículo.

En concreto se trata de una resolución de los Jurados de la ciudad de Zaragoza sobre un pleito que mantenían varios forjadores de espadas, y que se encuentra recogido en el Libro

\footnotetext{
13 Asso, 1798.

14 Ximenez de Embun, 1993: 141.

15 San Vicente Pino, 1988.
} 
de Actas de Zaragoza de $1549{ }^{16}$. Estos ciudadanos no eran otros que Julián del Rey de una parte, y su padre junto con su hermano por otra.

Atendido y considerado la question y diferencia que va y pende antte sus mercedes entre Julian del rey forjador de espadas de la una parte miguel del rey mayor y miguel del rey menor forjadores de espadas vezinos de la dicha ciudad...

La fecha de 1549 nos permite fijar la presencia de este espadero en un marco cronológico y geográfico concreto como es la Zaragoza de mediados del siglo XVI.

Los siguientes datos que nos aporta el documento son de carácter privado, ya que se refiere a un conflicto que se produce en el seno familiar y que permite documentar que Julián del Rey era hijo de Miguel del Rey mayor, y que Julián tenía un hermano Miguel del Rey menor. Los tres tenían el mismo oficio: forjar espadas.

En primer lugar esto parece terminar con la teoría según la cual Julián del Rey había adoptado ese nombre por haberle apadrinado Fernando el Católico. El hecho de que por lo menos una generación anterior lo portara con un nombre diferente, Miguel, parece desmentirlo.

El documento tiene como tema central la polémica cuestión de la marca que utilizó Julián del Rey, y que provoca la resolución de las autoridades municipales.

El símbolo que usaba Julián del Rey para identificar su obra era el que había heredado de su padre. El hermano de Julián, Miguel, apoyado por su padre, pretendía que también él pudiera usarla y el dicho miguel del rey mayor y menor padre e hijo pretendian el contrario.

Julián justifica su derecho al uso exclusivo de la marca basándose en tres puntos. Primero en que él había sido el primero en utilizarla a la hora de haberse examinado ante el gremio como espadero. Segundo por contar con el consentimiento de su padre, que fue el primero que la usó, y del que la heredó y con su voluntad. Y en tercer lugar por llevar mucho tiempo usándola por haverla hecho primero y mucho tiempo a.

De aquí se desprenden claramente dos cuestiones. Uno, el derecho de propiedad derivado del uso continuado. Y sobre todo el que fruto de ese uso en el tiempo, hubiera hecho famosa y buscada esa marca en el mercado espadero.

La última y más importante de las razones que alega es que las ordenanzas del oficio dictan que la marca que usaba un espadero no podía ser utilizada por ningún otro, y aun por obstarlo las ordinaciones del dicho officio. Decimos que quizás sea la más importante de las razones debido a que este pleito lo resuelven los Jurados, es decir una autoridad municipal. Al tratarse de casos relacionados con conflictos internos de un gremio, máxime cuando se esgrimen las ordenanzas gremiales como argumento, buscaban normalmente el asesoramiento de las autoridades gremiales ${ }^{17}$, havida informacion de los vehedores y examinadores del dicho officio y aun vistas las ordinaciones de aquel que hablan en este caso. De esta manera Julián del Rey se cargaba de razones al poner a las autoridades del gremio de su parte, al utilizar el texto de las ordenanzas de los espaderos de Zaragoza.

\footnotetext{
... la qual marca al tiempo que se examino tomo del dicho miguel del rey mayor padre suyo y con su voluntad que aquella haze agora el dicho miguel del rei menor hijo suyo pretendiendo aquella el no poder hazer por haverla el hecho primero y mucho tiempo a y aun por obstarlo las ordinaciones de su officio y el dicho miguel del rey mayor y menor padre e hijo pretendian el contrario...
}

Creemos que la lucha no por quitar la marca a Julián, sino por que la compartiera con su hermano, vendría dada por la fama que ya habrían adquirido las armas blancas que la portaban. No sabemos si la calidad de las piezas así marcadas tendría su origen en los conoci-

\footnotetext{
16 Archivo Municipal de Zaragoza. Libro de actos comunes de 1549, fol. 137v-138r.

17 Dueñas Beraiz, 1999: 99.
} 
mientos del padre, o si Julián del Rey fue el que a través de su habilidad en la confección de espadas la hizo tan preciada.

La marca en cuestión aparece claramente identificada en el texto como una cruz pequeña en los estoques, y una grande con metal colorado en las espadas.

... Julian del rey pretende que la marca que el haze en las espadas y otras cosas que de su officio haze que es una cruz pequeña en los estoques y grande en las espadas con metal colorado ...

El documento pone de relieve dos temas que nos gustaría señalar. En primer lugar la distinción, aunque sólo fuera en tamaño, de la marca dependiendo de la pieza, motivado quizá por la falta de espacio que en las hojas de los estoques existía, así como por la ausencia de recazo, lugar donde normalmente se encuentran dichas marcas.

En segundo lugar por la referencia que hace al color de la marca, con metal colorado, muy probablemente por estar rellenada con cobre como era usual en la época.

¿Cuál es la resolución final que se adopta respecto del uso de la marca? Los jurados fallaron a su favor, como era lógico tras la enumeración de todos los motivos expuestos por Julián para ser el único con derecho a utilizar las citadas marcas. Por lo tanto nadie más que Julián del Rey podía usarlas:

... oydas las dichas partes y havida informacion de los vehedores y examinadores del dicho officio y aun vistas las ordinaciones de aquel que hablan en este caso pronunciaron sentenciaron y declararon que el dicho miguel del rey menor ni mayor ni otra persona ni official alguno no puedan hazer ni contrahazer la dicha marca que el dicho Julian del rey haze ...

Esto se deba quizás a que el uso ilícito de las marcas de Julián del Rey no sólo se hacía por parte de su hermano ${ }^{18}$, sino que es probable de que con esta fórmula intentara evitar que nadie más se aprovechara de la fama de su marca.

Además aparecen dos términos, como contrahacer y contramarcas, que son prohibidos explícitamente por la decisión municipal. Creemos que no son sinónimos de hacer o marcar, sino que se refiere a la práctica de poner marcas conocidas sobre hojas que no tuvieran marcas, o que ya poseyeran otras de espaderos de menor importancia.

Las penas por no acatar esta resolución serían las existentes en las ordenanzas gremiales o del oficio.

y esto sopena de lo contenido en las dichas ordinaciones del oficio quien el contrario hiziere imposandoles sobre ello a los dichos miguel del rey mayor y menor padre e hijo silencio perpetuo etc. al qual se lo mandaron intimar etc. partes los dichos Julian del rey y miguel del rey su padre los quales açeptaron etc.

No contamos con las ordenanzas de espaderos de Zaragoza de la primera mitad del XVI, y por lo tanto no sabemos cuales serían exactamente estos castigos. La adición a las ordenanzas de espaderos de Zaragoza de $1602^{19}$, si permite suponer sin embargo que el castigo sería económico, unos cincuenta sueldos jaqueses, agravado por la pérdida de las espadas falsamente marcadas. Si se volviera a incurrir en ella se doblarían las penas anteriores.

Por desgracia para Julián del Rey estas penas no fueron suficientes para acabar con el uso fraudulento de su marca, tal y cómo lo refleja el último punto de las ordenanzas anteriormente citadas.

18 Resulta curioso observar como tampoco podía usar esas marcas el que había sido su dueño original Miguel del Rey mayor. Por lo tanto se ve el bien tan preciado que para un espadero era su marca. Y que una vez cedida no había manera de recuperarla, aunque fuera su hijo el nuevo poseedor.

19 AMZ., Libro de actos comunes, año 1602, fol. 276-277, en: SAN ViCENTE Op. Cit. pp. 41. 
... vendiendo assi mesmo espadas vizcaynas con cruzes coloradas por de Julian y con este color engañan a muchas personas con notable daño y perjuycio... ${ }^{20}$

\section{EL TEMA DEL «PERRILLO»}

La última cuestión a abordar sería el tema de la marca que se ha venido atribuyendo a este espadero. Tenemos que partir del hecho de que Palomares nunca indicó que dicha marca representara la figura de un perro. Fueron algunos de los autores que trataron el tema con posterioridad los que como tal la identificaron.

Pero, ¿por qué se interpretó la marca que Palomares atribuyó a Julián del Rey como un perro? Con independencia de que el dibujo puede interpretarse como tal, se debió seguramente a la abundante presencia de hojas de espadas de los siglos XVI-XVII, con representaciones de diferentes tipos de cánidos, que no suponen necesariamente una misma procedencia geográfica o cronológica. El conocimiento de espadas con este tipo de marcas por parte de la sociedad de la época tiene su reflejo en las abundantes referencias literarias castellanas contemporáneos a tales espadas ${ }^{21}$.

Tal cuestión mereció incluso la realización de un artículo hace unos años desde estas mismas páginas ${ }^{22}$, en el que se recogía la tradicional identificación de la marca atribuida a Julián del Rey, con las del resto de espadas con perros en sus hojas.

Si examinamos la figura que Palomares atribuye a Julián del Rey y la que muestran el resto de las hojas ${ }^{23}$, observaremos que son más las diferencias que las similitudes. Pero la diferencia más clara es que los otros «perros» que aparecen en las hojas de espadas suelen aparecer en actitud de correr o pasantes, mientras que la recogida por Palomares es rampante.

Según nuestra opinión se parte de un error de base. Este no es otro que el de considerar a la marca que Palomares atribuye a este forjador de hojas como un perro. La figura representa a un animal, si. Pero ¿cuál? Para saberlo es necesario observar detenidamente la forma con la que se nos presenta.

Cualquier aficionado a la heráldica conoce que esta postura, indicada al comienzo del presente artículo, se atribuye a unos pocos seres, entre los que no se incluyen los perros. En cambio si es típica en otros animales, como los caballos, ciervos, etc., pero sobre todo, y de manera destacada, en el caso del león.

Si partimos de la hipótesis de que se trata de un león, es cierto que no aparecen varios de los elementos significativos de estos animales como son sus melenas, las garras y el remate de la cola, pero también hemos de tener en cuenta para explicar estas ausencias que seguramente se trataría de una copia que Palomares hizo de una marca que aparecería en una hoja.

Imaginemos por un momento que se trate de un león ¿entonces la marca de Julián del Rey podría ser también la de un león?, ¿O quizás Palomares se equivocó? Creemos que la respuesta a ambas preguntas es negativa. Después de haber visto la información que sobre el respecto nos aporta el documento anterior podemos hallar una explicación que creemos bastante lógica.

Sabemos que además de las marcas de espaderos, solían figurar otras en algunas de las hojas. Éstas eran las que la ciudad tenía para marcar aquellas piezas que eran consideradas de calidad probada, contrastes, y que por lo tanto quedaban bajo la garantía y control de la ciudad.

\section{Ibidem.}

21 Son muchos los escritores españoles de la época que mencionan tales espadas. Uno de ellos es Cervantes quien la menciona en el episodio en el que Don Quijote obliga a soltar a un león que viajaba encerrado en una jaula, enfrentándose a él armado sólo con un espada del perrillo.

22 Rodríguez Lorente, 1964: 89-96.

23 Son abundantes las espadas con este tipo de imágenes en sus hojas. Real Armería de Madrid: G. 45; G. 101 y G. 116. Wallace Collection: A 484; A529 y A576. Royal Arms at Rosemborg No 14 y 30, etc. Normalmente asociadas al célebre lobo o perro de Passau y Solingen. 
portenox dela prite condenamos no. obstante lo allegado deduzido $\partial$

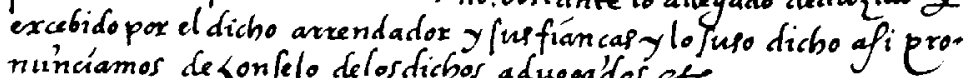
nincíamos de jonfelo delostichos aduogaldos et ofaiglulo go alfonfus laqial somarypronunciación ofidada y promilgada pox los

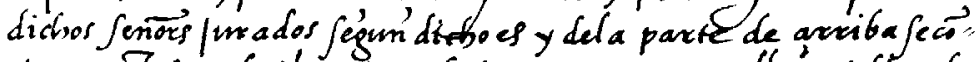
tione prites ambas las fuso dichas partes y aquella's pidiendo proninciar ete os incotineti los dirfor / exorimo prat domings Destaraq I Glas nanarero pro cuñàoves del dicho terio deal mo"

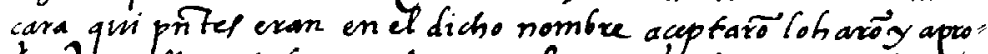

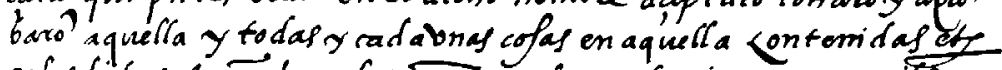

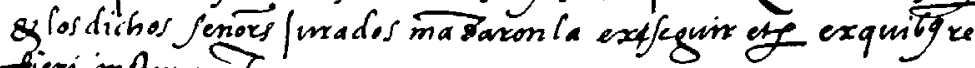
fievi mArumefum $=-$

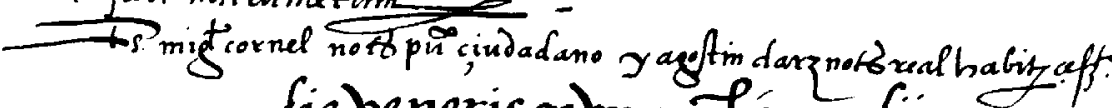
die beneris $x$ by mejismadij jindomigy pontiscebagu/te

hedorcs de? merecererof

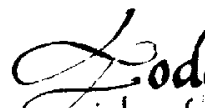

\section{odenzizé los}<smiles>C1C[C@H]2CC[C@H]12</smiles>

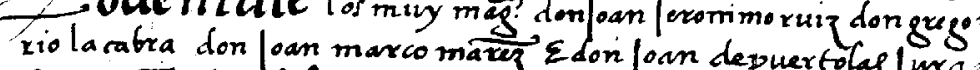
dos a pintacion delos mayordom don loan depuertolas i $u x$ : cirdad oxearon y robrazon en behedores delos dichos delaficha

Seriademarens de ejpadas.

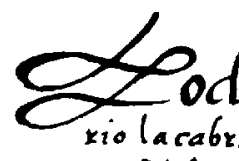

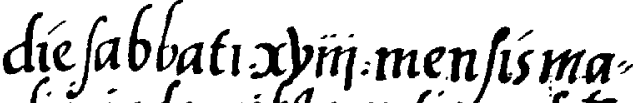 dij. indomiby pontis ce $\beta^{t_{2}}$.}

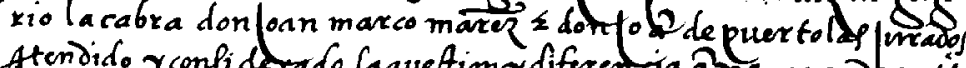
Atonido Yconfiderado raqueftion ydiforentia ara sus mercedep entre wian del rey for ador de epadas dela

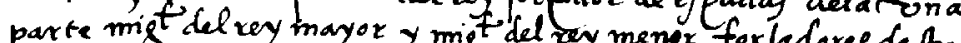
pupres mex fod oves de pa. das voezinos deladicha ciudad tela pancte otra en 2 pore que richo fuction del rey pretende quel a maxra oel haze enlas opa"

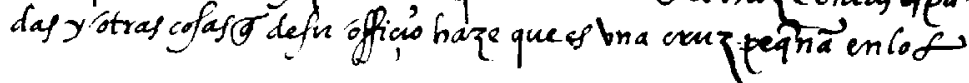

Texto original del pleito de Julián del Rey con su padre y hermano existente en el Archivo Municipal de Zaragoza. Libro de actos comunes, año 1602, fol. 276-277 


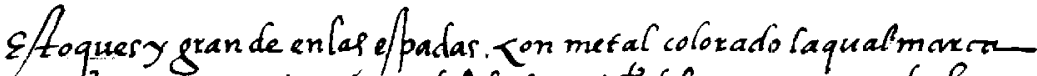
altpo quefe oxamino tomo del dicho migt' del rey mayor padrefingo y confu volum fad 9 a quella haze agora el dicho mi to del reimonor

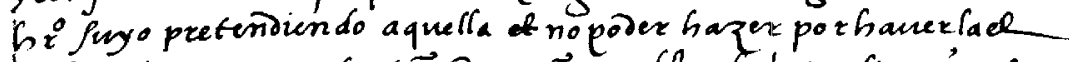
hechoprimexoy muchotpo@yañ por obtarto las ordina ciones

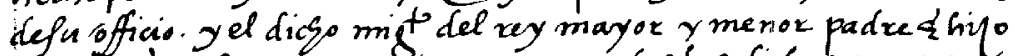
pretendian el contraxio portanto oydas las dich os partes $\frac{1}{2}$ havida informacion defos rochedoref y examinadoces tel dicso officio y aum biftag las ordina ciones deaquel $\vec{q}$ hablan enetterifo promiciaron femavon declaxano 9 eldics m in del xey menor

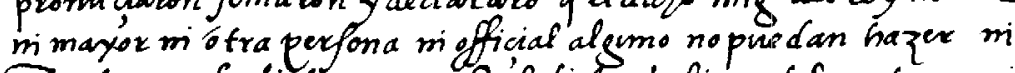
cotra bazer ladicha maxra 9 él dicho julian def rey haze $m$ menos con ponexle Etra maxca mo que a quella fol amete haga el

dicgo Iutian del rey for a dox como arriba dicto s yefto fopona

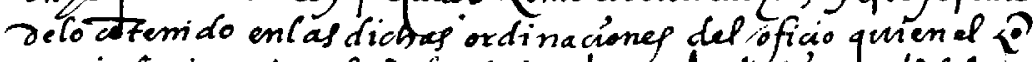

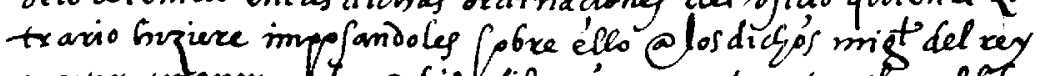

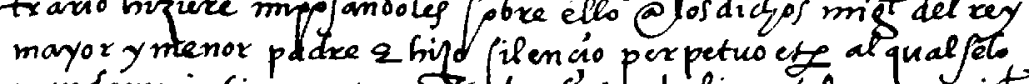
mandaren intimax eff pntolosdigos ulian del rey y migt

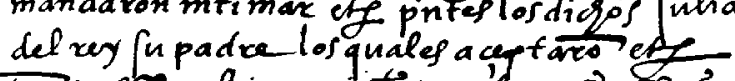

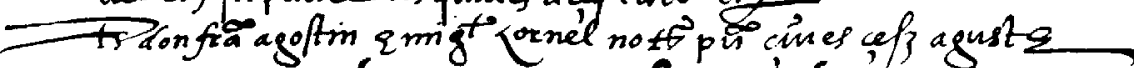

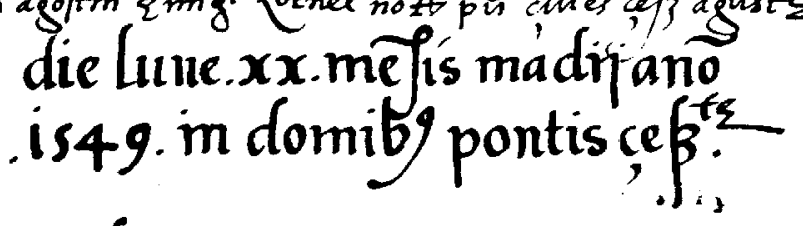

Bodem die en pracia delos muy mag don loan leronimo ruiz dongregorio la cabra donjoan marco maver mire aggtm de gu" rrea $y$ don oan depuertolas tuxados parecí anton de ra moratior

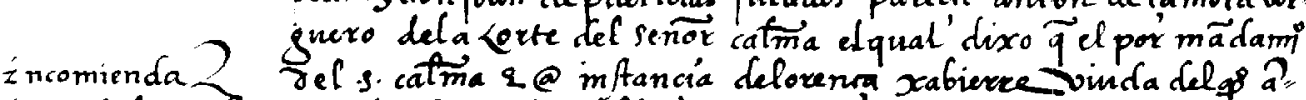

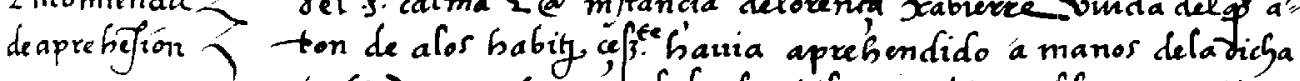
Lerfe on guerto con axboles frutiferes $Y$ tierra blan ra contiguos lite en mosnillo terio dela didsa ciudad que es cimo robas detié rra posomasio menos $\bar{q}$ a fructa' <enguerto déageftin del pon con campo de peoro gxan y con compo de doningoxpoual $B$ afi dixo que feleg en oomentina y en comendo ege glos dichos fe. noxs juxados dixeron Gaxian loq deuian ete it enomedaxonlo @onluras perez déolitian mayordomo a qual lo mandaron intimax etfe

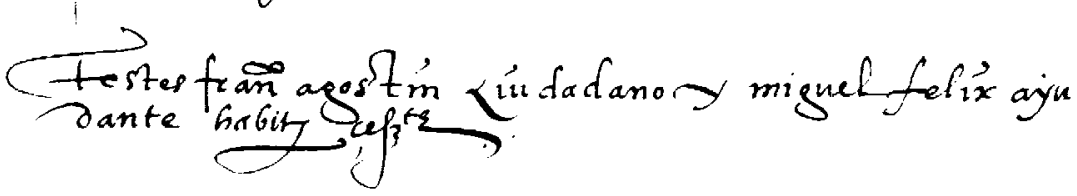

Texto original del pleito de Julián del Rey con su padre y hermano existente en el Archivo Municipal de Zaragoza. Libro de actos comunes, año 1602, fol. 276-277 
Fue frecuente el uso de los símbolos heráldicos de la ciudad como marca de la ciudad. Y eran muchos los escudos de ciudades españolas de la época que presentaban leones, hasta incluso la actualidad. Aunque si las comparamos con las de las ciudades donde en un principio se localizó a nuestro hombre obtendremos una sola, Zaragoza.

Esto es sólo una teoría y por lo tanto es necesario aportar datos sobre el uso del león como marca de garantía de la ciudad por los artesanos zaragozanos. Los espaderos no dicen nada sobre la forma de la marca que la ciudad colocaba en sus espadas como garantía de calidad $^{24}$. En cambio si que lo hace un gremio tan afín como el de los cuchilleros ${ }^{25}$, en una fecha tan temprana como 1423.

Las ordenanzas de los cuchilleros zaragozanos de $1423^{26}$, entre los diferentes puntos que componen éste interesante documento, encontramos dos, nueve y once ${ }^{27}$, en los cuales se trata el tema de la marca que los puñales hechos en la ciudad debían llevar además de la del cuchillero. El primero obliga a todos los cuchilleros de la ciudad a poner la marca de la ciudad en sus obras. Y en la segunda se prohibe que las obras realizadas fuera de Zaragoza, portaran dicha marca. En ambos puntos se dice que la marca, senyal, debía de ser la del león. Con lo cual tenemos una conexión clara entre la marca del león en las armas blancas y la ciudad de Zaragoza.

E qualquiere que vendera punyal o punyales sines de seyer meso el senyal del leon, encorra por cada punyal en pena de $\mathrm{V}$ sueldos, divididera ut supra. Empero que qualquiere cuytillero por si pueda meter el dito senyal de leon pues que por los ditos vehedores seran ya reconocidos.

Por todo ello, creemos posible que Palomares, al igual que hizo en otras ocasiones, localizara esa marca en una hoja firmada por Julián del Rey, atribuyéndosela erróneamente como la personal en lugar de cómo la de contraste de Zaragoza.

\section{CONCLUSIONES}

El documento no puede ser más claro e ilustrativo sobre casi todos los aspectos que no eran conocidos o que no estaban claros en el pasado. Julián del Rey trabajó en Zaragoza en la primera mitad del siglo XVI. La marca que utilizó fue la de una cruz con cobre embutido en las espadas, y otra más pequeña en los estoques.

Existe una posible marca de Julián del Rey, ya que alrededor de ella aparece su nombre $^{28}$, y que además concuerda en la forma con la que aparece en este documento. Está for-

24 Dueñas Beraiz Op. Cit. pp. 108.

25 Entiéndase aquí el término cuchillero cómo fabricante armas blancas cortas. Especialmente puñales ya que este es el término utilizado en el documento.

26 Falcón Pérez, 1977: 59-77.

27 [9] Item, quieren e ordenan que en qualesquiere punyales que se acabaran en la dita ciudat, sean tenidos qualesquiere menestrales de posar e meter, posen e metan el senyal de la dita ciudat, ultra el senyal que fara cada un cuytillero, el qual senyal yes un leon; e que ninguno no pueda vender punyal o punyales sino que sea senyalado del dito senyal por que aquestos tales pueden seyer dados por buenos e mercadores. E qualquiere que vendera punyal o punyales sines de seyer meso el senyal del leon, encorra por cada punyal en pena de V sueldos, divididera ut supra. Empero que qualquiere cuytillero por si pueda meter el dito senyal de leon pues que por los ditos vehedores seran ya reconocidos.

[11] Item, quieren e ordenan que ningun cuytillero, ni usant del officio por toda la senyoria del regno de Aragon, no pueda meter otro tal senyal de leon como yes el de la dita ciudat en punyal o punyales algunos, por tal que muytos en otras ciudades, villas, e estruenta farian e estruenta facen el dito senyal e dicen que son de la ciudat de Çaragoça, por do enganyan e deciben la gent, lo qual redunda en grant dayo de la cosa publica. E qualquiere cuytillero de fuera de Çazaroça que tal senyal fara o posara en punyal alguno, pierda la dita obra e haya de pena cincientos sueldos pora los cofres del senyor rey, e la obra pierda.

28 Boeheim, 1897. 
mada por una cruz dentro de un escudete coronado, que a su vez está rodeado por un círculo en el que va escrito el nombre de Julián del Rey. Si fuera auténtica o una «fiel falsificación $^{29}{ } \gg$ de la época, la cruz a la que el documento se refiere sería una cruz griega.

La espada a la que pertenece dicha marca se encuentra en el Museo de l'Armée de París (G-120) ${ }^{30}$, y nos ofrece todas las garantías sobre su autenticidad, por lo que se vería confirmada la forma exacta de la marca utilizada por Julián del Rey, y motivo del pleito.

Una cuestión sobre la cual no se aporta mayor información en el presente documento, es la de su condición de morisco. Si conocemos, que su apellido era uno de los adoptados frecuentemente por los de la comunidad morisca ${ }^{31}$. Es de sobra conocida la fuerte presencia de esta población en la Zaragoza de aquella época.

La presencia de esta comunidad como fabricantes de armas, escopetas, puñales y sobre todo espadas, en la capital aragonesa se encuentra también documentada en una comunicación que el consistorio de Zaragoza remitió a Carlos V en 1525 sobre los daños que una posible expulsión de los moros de Aragón tendría sobre este tipo de producción ${ }^{32}$.

Sabemos en cambio que existen diversas referencias tanto en inventarios como en la literatura de la época, al moro de Zaragoza, y su identificación de tal personaje con Julián del Rey. Esta identificación se ve cuestionada al menos en parte por los datos que aporta el inventario de la Real Armería de $1594^{33}$.

En él se enumeran dos piezas de la siguiente manera:

otra oja de espada corta del moro de Zaragoza.

otra oja de espada de su hijo del Moro de zaragoza Julian

Son varias las conclusiones que se pueden extraer de esta pequeña relación. En primer lugar que ambos forjadores de espadas gozaban de una gran reputación en aquella época. Nos basamos para realizar esta afirmación en el hecho de que se utiliza como elemento que las identifica y valora, la referencia a sus creadores, usando el apelativo del primero, y el nombre y parentesco del segundo. Este sistema se utiliza en primer lugar siempre que se disponga de dicha información, y segundo en el caso de tratarse de piezas importantes, y este parámetro lo aporta básicamente la fama del artesano que la ha realizado.

Al mismo tiempo, que se denomine al primero de esa manera, parece indicar que las numerosas referencias que sobre el moro de Zaragoza se realizaron en el pasado ${ }^{34}$ fueran referidas a Miguel del Rey mayor, y no a su hijo Julián.

Nos atrevemos a pensar incluso que la variación que de esta forma de definirlo existía, morillo de zaragoza $a^{35}$, haría referencia a Julián del Rey usando el diminutivo para distinguir al padre, Miguel, del hijo, Julián. Por último también sirve para demostrar una vez más la vinculación de ambos personajes con la ciudad de Zaragoza.

Quién sabe si él y sus descendientes fueron víctimas de las posteriores expulsiones de moriscos que posteriormente se producirán en la corona española, la primera tras la subleva-

29 Con este término aparentemente contradictorio nos referimos a una espada que sin ser hecha por Julián del Rey, el espadero que la falsificó realizó tan bien su trabajo que incluso copió exactamente la marca de una espada original, con lo cual la forma de la marca sería la correcta.

30 Debo la obtención de este dato a la amabilidad y erudición del Dr. Don José A. Godoy, conservador del Musée d'Art et Histoire de Ginebra.

31 Incluso existía otro espadero morisco en Sevilla, llamado Gaspar de los Reyes de León, en: Leguina Op. Cit. pp. 143.

32 Asso. Op. Cit. pp.130

33 Inventario de la Real Armería de 1594, fol. 70 rº. Quiero agradecer la obtención de dicha información a su conservador el Dr. Don Alvaro Soler del Campo.

34 Valencia de Don Juan. Op. Cit. pp. 194

35 Asso. Op. Cit. 
ción de 1568-1570, y la general de 1610, lo cual explicaría en parte los escasos datos que sobre ellos tenemos.

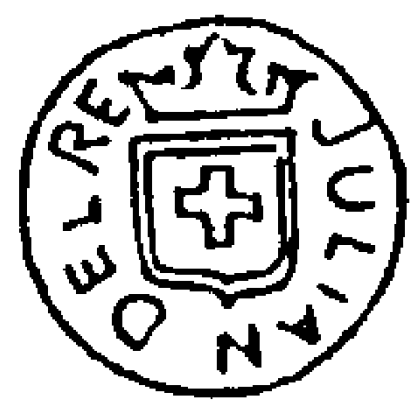

Fig. 3. Marca de la espada G.120 del Musée de l’Armée atribuida a Julián del Rey.

Si parece claro que Julián del Rey es ya un espadero con experiencia en 1549 tal y como indica al hacer valer su derecho a la marca de su padre, ...no poder hazer por haverla el hecho primero y mucho tiempo a ..., tal y como vimos anteriormente. Por lo tanto, su padre habría desarrollado su labor artesanal fundamentalmente durante el primer cuarto del siglo XVI. Con lo cual la existencia del Julián del Rey al servicio del último rey moro, antepasado de éste, a pesar de no ser imposible, es poco probable. En cualquier caso, la cronología indica que su padre Miguel mayor, sería el que podría haber trabajado tanto para Boabdil como para Fernando el Católico.

A pesar de que todavía son muchos los aspectos desconocidos sobre este maestro de forjar hojas de espadas, si se han podido clarificar al menos algunos hechos de su vida.

En primer lugar la ubicación cronológica y geográfica de Julián del Rey, la Zaragoza de mediados del XVI; que se trataba del representante de por lo menos una segunda generación de excelentes fabricantes de armas blancas, formada de al menos su padre Miguel mayor y de su hermano Miguel del Rey menor; que las marcas usadas por él y su padre eran las mismas y se trataba de cruces pequeñas en los estoques, y más grandes rellenas de cobre en las espadas; y que parece más que probable su condición de morisco, siendo llamado el padre el moro de Zaragoza, y el morillo su hijo Julián.

El no conocer con seguridad la forma de la cruz que hacía, a pesar de la existencia de la espada del Musée de l'Armée, y el hecho que sea una marca bastante utilizada tanto dentro como fuera de nuestro país, hace muy difícil la identificación de espadas por él templadas. La localización de más espadas con marcas similares a la existente en París, marca acompañada de su nombre, podría indicarnos con mayor seguridad que esta fue la que utilizó este espadero.

A pesar de todo, Julián del Rey seguirá siendo uno de los más afamados y misteriosos de los artesanos hispanos que en la Edad Moderna se dedicaron a templar espadas. 


\section{APÉNDICE}

Archivo Municipal de Zaragoza. Libro de actos comunes, año 1602, fol. 276-277

Sentencia de marcas de espadas (al margen)

Eodem die los muy magnificos don Joan Jeronimo ruiz don gregorio cabra don joan marco martinez e don juan de puertolas jurados Atendido y considerado la question y diferencia que va y pende antte sus mercedes entre Julian del rey forjador de espadas de la una parte miguel del rey mayor y miguel del rey menor forjadores de espadas vezinos de la dicha ciudad de la parte otra en el sobre que el dicho Julian del rey pretende que la marca que el haze en las espadas y otras cosas que de su officio haze que es una cruz pequeña en los estoques y grande en las espadas con metal colorado la qual marca al tiempo que se examino tomo del dicho miguel del rey mayor padre suyo y consu voluntad que aquella haze agora el dicho miguel del rei menor hijo suyo pretendiendo aquella el no poder hazer por haverla el hecho primero y mucho tiempo a y aun por obstarlo las ordinaciones de su officio y el dicho miguel del rey mayor y menor padre e hijo pretendian el contrario por tanto oydas las dichas partes y havida informacion de los vehedores y examinadores del dicho officio y aun vistas las ordinaciones de aquel que hablan en este caso pronunciaron sentenciaron y declararon que el dicho miguel del rey menor ni mayor ni otra persona ni official alguno no puedan hazer ni contrahazer la dicha marca que el dicho Julian del rey haze ni menos con ponerle contramarca sino que aquella solamente haga el dicho Julian del rey forjador como arriba dicho es y esto sopena de lo contenido en las dichas ordinaciones del oficio quien el contrario hiziere imposandoles sobre ello a los dichos miguel del rey mayor y menor padre e hijo silencio perpetuo etc. al qual se lo mandaron intimar etc. partes los dichos Julian del rey y miguel del rey su padre los quales açeptaron etc.

Testes Don francisco agostin e miguel cornel notario publico çives cesarauguste

\section{AGRADECIMIENTOS}

Quisiera agradecer la valiosa ayuda e indicaciones que me han prestado el Dr. Don Alvaro Soler del Campo, conservador de la Real Armería de Madrid, y Don José A. Godoy conservador del Musée d' Art et d'Histoire de Ginebra, para la realización de este artículo.

GERMÁN DUEÑAS BERAIZ

C/ Savia 3, 3 B - 16004 Cuenca

\section{BIBLIOGRAFÍA}

Asso, I. (1798): Historia económico-política de Aragón. Zaragoza.

BEAUMONT, E. (1885 ): La fleur de belles epées. París.

BoEHEIM, W. (1897): «Meister der Waffenschmiedekunst vom 14. bis ins 18». Ein Beitrag zur Geschichte der Hunst und des Kunsthandwerks XI, 246 S.XX Taf.. W. Moeser.

DueÑas BeRAIZ, G. (1999): Los gremios de espaderos españoles en la Península Ibérica (S. XVIXVII). Aproximación a su estudio (Grado de Salamanca inédito). Departamento de Historia Moderna, Medieval y Contemporánea. Universidad de Salamanca.

FALCON PEREZ, Mª I. (1977): «La cofradía de cuchilleros zaragozanos en el siglo XV. Las ordenanzas de 1423.». Homenaje a Don José María Lacarra IV: 59-77.

LEGUINA, E. (1897): Los maestros espaderos. Sevilla.

LEGUINA, E. (1912): Glosario de voces de armería. Madrid. 
MAINDRON, (1890 ): Les armes. París.

MARTINEZ Romero, A. (1849): Catálogo de la Real Armería mandado formar por S.M. siendo director general de reales caballeriza, armería y yeguada, el Excmo. Señor Don José María Marchesi. Impr. Aguado. Madrid.

RODRIGUEZ LORENTE, J.L. (1964): «La marca del perrillo del espadero español Julián del Rey», Gladius III: 89-96.

SAN VICENTE PINO, A. (1988): Instrumentos para una historia social y económica del trabajo en Zaragoza en los siglos XV-XVIII. Real Sociedad Económica Aragonesa de Amigos del País. Zaragoza.

Valencia de Don Juan, Conde Viudo de [Juan Bautista Crooke y Navarrot] (1898): Catálogo Histórico-Descriptivo de la Real Armería de Madrid. Real Casa. Madrid.

Ximenez De Embun, T. (1993): Descripción histórica de la antigua Zaragoza y de sus términos municipales. Librería París-Valencia (Reed). Valencia. 